OPEN

SUBJECT AREAS:

OPTICAL PROPERTIES AND

DEVICES

RAMAN SPECTROSCOPY

Received

15 August 2013

Accepted

8 November 2013

Published

26 November 2013

Correspondence and requests for materials should be addressed to iisc.ernet.in)

\section{S.U. (umapathy@ipc.}

\title{
Is Chemically Synthesized Graphene 'Really' a Unique Substrate for SERS and Fluorescence Quenching?
}

\author{
Sanchita Sil ${ }^{1,2}$, Nikki Kuhar' ${ }^{1}$ Somnath Acharya' \& Siva Umapathy ${ }^{1}$
}

\begin{abstract}
'Department of Inorganic \& Physical Chemistry, Indian Institute of Science, Bangalore, India, ${ }^{2}$ High Energy Materials research Laboratory, Sutarwadi, Pune, India.
\end{abstract}

We demonstrate observation of Raman signals of different analytes adsorbed on carbonaceous materials, such as, chemically reduced graphene, graphene oxide (GO), multi-walled carbon nanotube (MWCNT), graphite and activated carbon. The analytes selected for the study were Rhodamine 6G (R6G) (in resonant conditions), Rhodamine B (RB), Nile blue (NBA), Crystal Violet (CV) and acetaminophen (paracetamol). All the analytes except paracetamol absorb and fluoresce in the visible region. In this article we provide experimental evidence of the fact that observation of Raman signals of analytes on such carbonaceous materials are more due to resonance effect, suppression of fluorescence and efficient adsorption and that this property in not unique to graphene or nanotubes but prevalent for various type of carbon materials.

$\checkmark$ )

raphene, the most recently discovered allotrope of carbon, also termed as a wonder material and given many adjectives has been a source of intrigue amongst researchers all over the world owing to its unique electronic structure ${ }^{1}$. It is considered to be the building block of other carbon allotropes, such as, graphite, nanotubes, buckyballs, and others ${ }^{2}$. The unique chemical, electrical and mechanical properties of graphene can be attributed to the zero band gap and $2 \mathrm{D}$ honey-comb structure ${ }^{3}$.Among many applications of graphene, which include electrochemical sensors, field effect transistors, chemical catalyst, energy storage and conversion devices etc. ${ }^{4}$, one of the promising applications is its use as a surface enhanced Raman spectroscopic (SERS) substrate and a suitable alternative for noble metals ${ }^{5-9}$. Electromagnetic (EM) enhancement on the surface of graphene has been negated as its surface is relatively smooth and optical transmission through its surface is greater than $95 \%{ }^{10}$. Furthermore, the surface plasmon on graphene is in the range of terahert $\mathrm{z}^{11,12}$ rather than in the visible range therefore, unlikely to be excited using visible wavelength. Hence the enhancement in Raman signals observed in graphene has been attributed to chemical enhancement mechanism, fluorescence rejection and/or efficient adsorption ${ }^{6,13,14}$.

Although quenching of fluorescence by metals has been known in the optical community for several dec$\operatorname{ades}^{15,16}$, Xie et al., for the first time, reported fluorescence suppression by graphene using resonance Raman spectroscopic studies of rhodamine $6 \mathrm{G}$ (R6G) and protoporphyrin IX (PPP) on graphene ${ }^{13}$. The same authors in their subsequent publication ${ }^{5}$ reported enhancement of Raman signal of analytes by graphene and coined the phrase graphene enhanced Raman spectroscopy (GERS). They observed that single layer graphene provided Raman enhancement with many analytes owing to the chemical enhancement mechanism and also as the number of layers were increased, the Raman intensities decreased and no signal could be observed on graphite or highly oriented pyrolitic graphite $(\mathrm{HOPG})^{5}$. It was concluded that the interference effect induced by the different numbers of graphene layers does not make a major contribution to the observed intensity ${ }^{5}$. However, more recently it has been reported ${ }^{17}$ that GERS is independent of the layer thickness and Raman enhancement can be observed up to six layer graphene thickness. In contrast, Thrall et al. ${ }^{18}$ have reported the observation of a threefold reduction in the Raman enhancement and concluded that the strong quenching of R6G fluorescence was responsible for the sensitive Raman detection of the molecule.

In addition, popular carbon materials like fulleren $e^{19}$, carbon nanotubes $^{20}$, graphene oxide ${ }^{21}$, reduced graphene oxide with covalently linked $\beta$-cyclodextrin ${ }^{22}$ and graphene ${ }^{23}$ have been shown to quench fluorescence of aromatic compounds either via electron transfer or energy transfer. Theoretical studies on the distance dependence of the rate of energy transfer from a dye molecule to a graphene sheet have also been reported ${ }^{24,25}$. Such quenching of fluorescence does produce good Raman signals which sometimes have been interpreted as SERS. Kagan and McCreery as early as 1994, reported the observation of Raman spectra of normally fluorescent compounds such as 

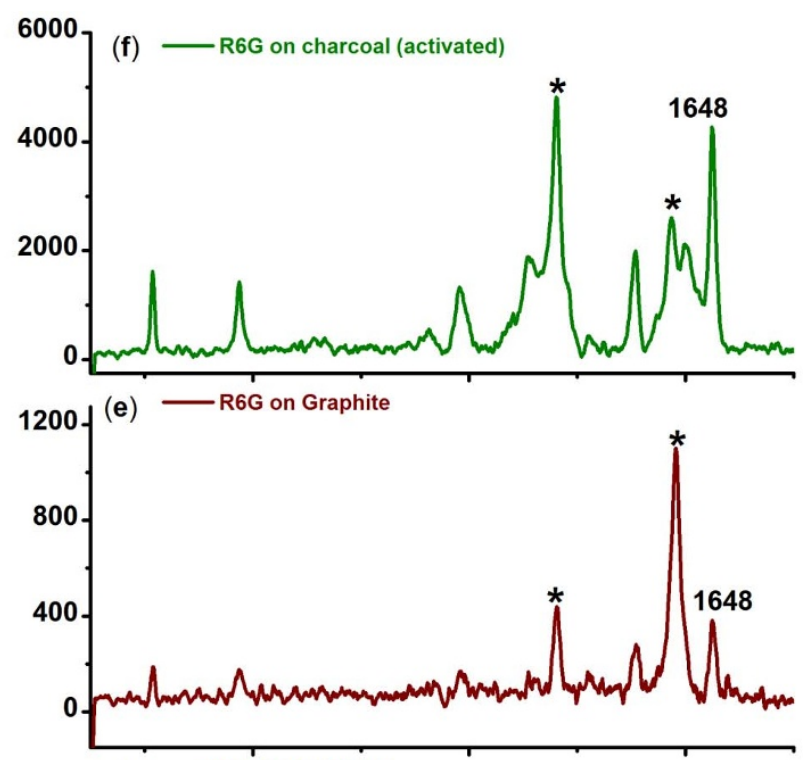

(d) (R6G on MWCNT)
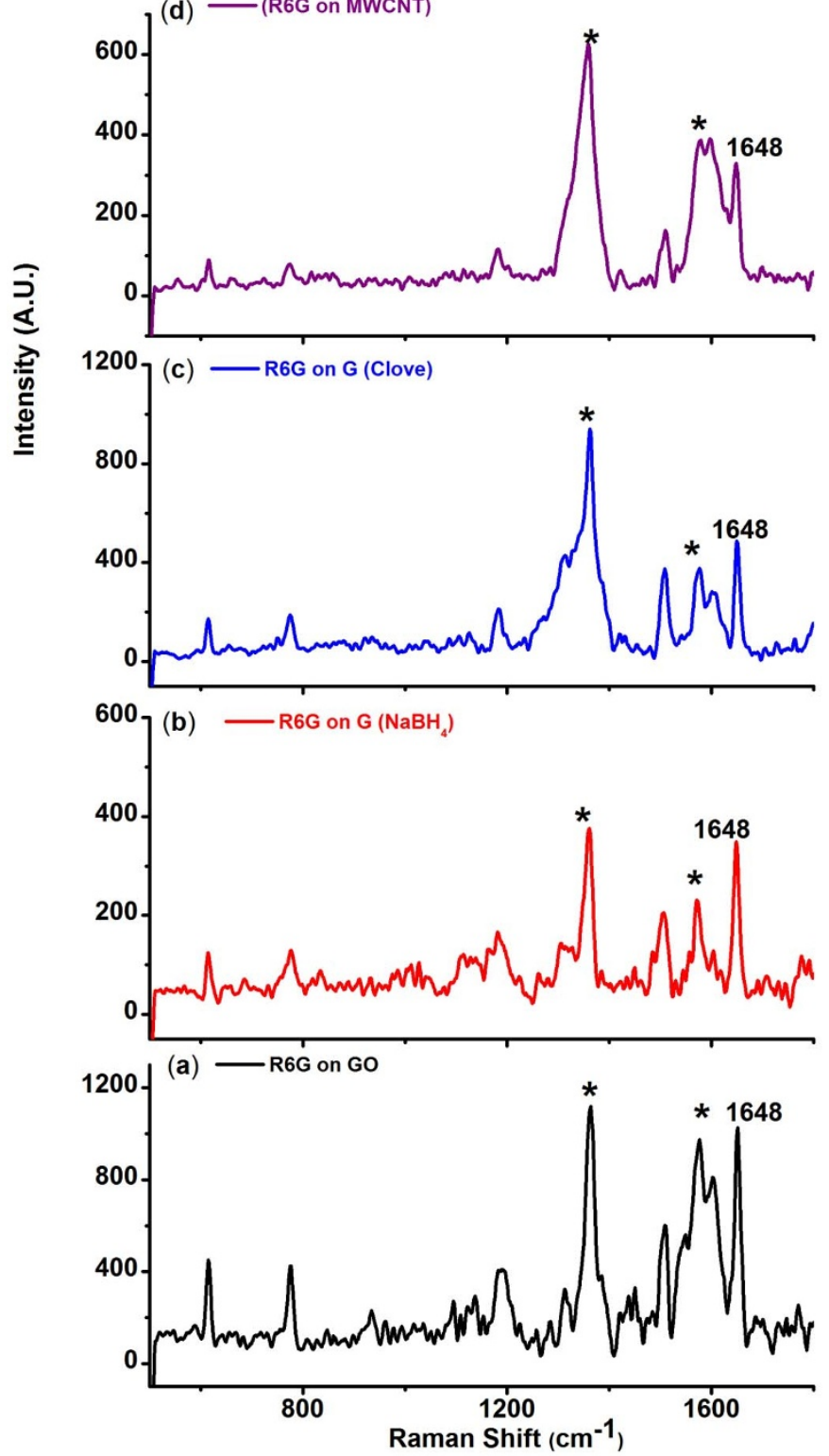

Figure $1 \mid$ Raman spectra of $10^{-7} \mathrm{M}$ rhodamine 6G (R6G) adsorbed on (a) Graphene oxide, (b) graphene reduced by $\mathrm{NaBH}_{4}$, (c) graphene reduced by clove extract, (d) multi-walled carbon nanotube (MWCNT), (e) graphite and (f) charcoal (activated). (* represent carbon peaks, $1648 \mathrm{~cm}^{-1}$ peak corresponds to R6G).

Rhodamine 6G (R6G), IR125, bis(methylstyry1)benne(BMB) and fluoranthene adsorbed on $\mathrm{sp}^{2}$-hybridized carbon rod as carbon substrate $^{26}$ and they also attributed their observation to quenching of fluorescence and good adsorption.

Herein, we demonstrate the observation of Raman signals of different analytes adsorbed on different type of carbonaceous materials, such as, chemically reduced few layer graphene, graphene oxide (GO), multi-walled carbon nanotube (MWCNT), graphite and activated charcoal. The objective is to provide experimental evidence of the fact that a) observation of Raman signals on such carbonaceous materials are more due to resonance Raman effects ${ }^{26,27}$, suppression of fluorescence and efficient adsorption as a solid (substrate) and b) observed enhanced Raman signals under resonant conditions for fluorescent compounds is not unique to graphene or nanotubes but prevalent for any type of carbon material. In addition, this study would also form the basis for considering other carbon substrates which are available in abundance and are cost-effective for the study of Raman enhancements in the future.

\section{Results}

Chemically synthesized graphene (few layer graphene) along with other carbon based materials, such as, graphene oxide, graphite, multi-walled carbon nanotubes (MWCNT), and activated charcoal were chosen as substrates to explore the possible Raman enhancement from analytes which are fluorescent (coloured) as well as nonfluorescent (white). Graphene oxide (GO) was synthesized in the laboratory from graphite powder following the modified Hummers method $^{28,29}$. Graphene was prepared by chemically reducing GO using $\mathrm{NaBH}_{4}{ }^{30}$ and clove extract [Details of the characterisation of graphene are provided in Supplementary Information]. Detailed characterization was carried out using UV-Vis, IR, XRD, and Raman spectroscopy to confirm the formation of clove reduced graphene. Raman spectroscopy has generally been used to characterize the formation of graphene and the number of layers $s^{7,9,31,32}$, as is the case in the present experiments (Supplementary Information S2a-e). Surface area measurements were also carried out for the carbon based materials. The BET surface area along with pore diameters has been provided in table $\mathrm{S} 4$ in the supplementary information.

All the analytes (Rhodamine 6G, Rhodamine B (RhB), Crystal Violet (CV), Nile Blue A (NBA) and paracetamol, Figure S3) selected for the study, except paracetamol (S3), strongly fluoresce in the visible region. Hence, the analytes were probed with the excitation wavelength of $514.5 \mathrm{~nm}$ which was close to the absorption maxima of R6G [(527 nm (water), supplementary information, S5a] and RhB [542.8 $\mathrm{nm}$ (water), supplementary information, S5b]. The wavelength was chosen specifically to understand the effect of interference from fluorescence on the Raman spectrum of these molecules when adsorbed on carbon substrates. Solid R6G shows Raman signal at $614,774,1187,1362,1507,1571$ and $1648 \mathrm{~cm}^{-1}$ respectively with $514.5 \mathrm{~nm}$ laser source (supplementary information, S6). The peaks obtained are in good agreement with earlier reports on Raman and resonance Raman spectra of $\mathrm{R} 6 \mathrm{G}^{33,34}$. The $614 \mathrm{~cm}^{-1}$ peak corresponds to the $\mathrm{C}-\mathrm{C}-\mathrm{C}$ ring in-plane bending mode. The $\mathrm{C}-\mathrm{H}$ outof-plane bending mode for R6G is observed at $774 \mathrm{~cm}^{-1}$ while the $\mathrm{C}-\mathrm{O}-\mathrm{C}$ stretching frequency appears at $1187 \mathrm{~cm}^{-1}$. Peaks centered at $1362,1507,1571$ and $1648 \mathrm{~cm}^{-1}$ are attributed to the aromatic C-C stretch of the R6G molecule ${ }^{35}$. We would like to note that the normal (without any carbon substrates) Raman spectrum of this molecule in solution is not discernible owing to fluorescence. However, from a drop of the same solution on adsorption on the carbon substrates, Raman spectra were recorded for $10^{-7} \mathrm{M}$ R6G on different carbon substrates as shown in figure 1. Furthermore, the Raman experi- 
ments for all the substrates and analytes were conducted under similar conditions. The concentration of the analyte was constant for all the carbon substrates. For instance, a 10 ul of $10^{-7} \mathrm{M}$ of the concentration of an analyte contains $\sim 10^{11}$ molecules. The number of molecules contained in the focal volume using a long working distance microscope objective $50 \times \mathrm{L}$ would be $\sim 10^{5}$.

A comparison of the surface area along with the variation of the intensity of the $1648 \mathrm{~cm}^{-1}$ peak for R6G at $10^{-7} \mathrm{M}$ concentration for all the carbon substrates is depicted in figure 2. The surface area measurements also revealed an increase in surface area from chemically reduced graphene to activated charcoal. At a first glance it would rather appear that there exists a trend on comparing the Raman signals with the surface area values, which is observed in the case of charcoal. However, at a closer inspection it is apparent that surface area alone, cannot explain the observation of the Raman signals observed for R6G adsorbed on the other carbonaceous substrates such as GO, MWCNT, chemically reduced graphene and graphite. Other mechanisms such as quenching of fluorescence and resonance effects could also be contributing factors for such enhancement. Based on this observation, further experiment for $10^{-9} \mathrm{M}$ concentration of R6G on graphene and charcoal (activated) was also performed (Figure 3). In order to avoid the overwhelming intensity of the carbon bands ( $G$ and $D$ bands), the Raman spectra were obtained at lower wave number region (up to $1200 \mathrm{~cm}^{-1}$ ). Raman spectra of R6G at this concentration for both chemically reduced graphene as well as charcoal (activated) could be clearly recorded. The observed Raman peaks matched well with the solid state R6G spectrum. The Raman enhancement on the basis of the intensity counts in the case of charcoal (activated) was almost five times more than graphene for all the peaks. Experiments on other analytes such as RhB, CV and NBA have also been conducted. Raman spectra could be obtained for the analytes CV and NBA at $10^{-6} \mathrm{M}$ concentrations and at $10^{-7} \mathrm{M}$ concentration for $\mathrm{RhB}$ on different carbon substrates (see supplementary information, S7ac). It should be noted that the Raman signals for CV and NBA was relatively weaker as the excitation wavelength $(514.5 \mathrm{~nm})$ was not in direct resonance with the absorption of $\mathrm{CV}$ is $587 \mathrm{~nm}$ (water) and NBA is $635 \mathrm{~nm}$ (water) (supplementary information, S5c-d).

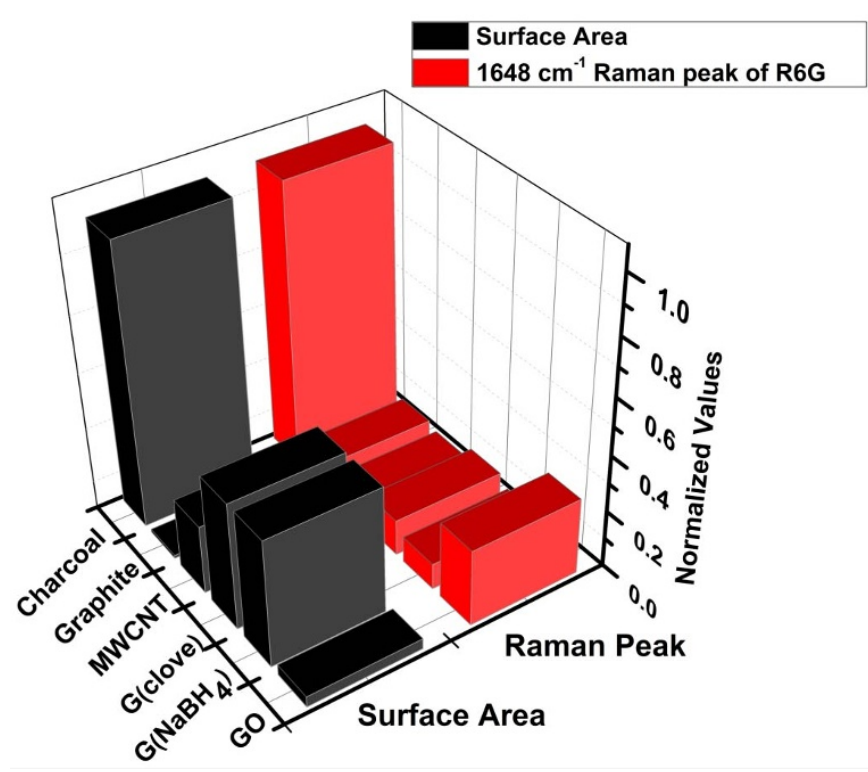

Figure 2 Variation of BET surface area and Raman spectra of the $1648 \mathrm{~cm}^{-1}$ peak for $10^{-7} \mathrm{M}$ rhodamine 6G (R6G) adsorbed on (a) GO, (b) graphene reduced by $\mathrm{NaBH} 4$, (c) graphene reduced by clove extract, (d) multi-walled carbon nanotube (MWCNT), (e) graphite and (f) charcoal (activated).

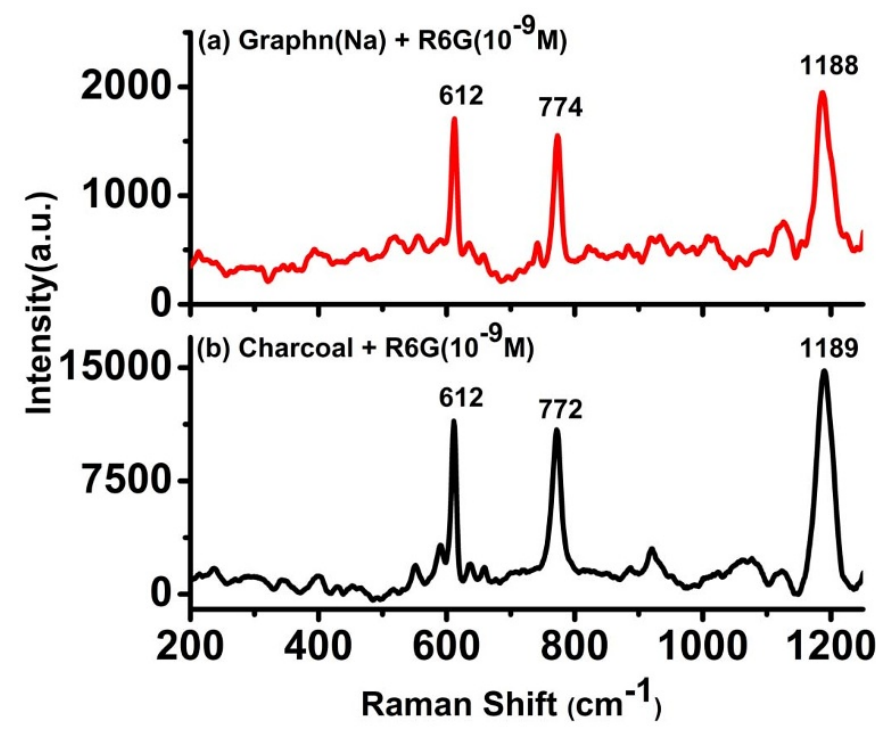

Figure 3 Raman spectra of $10^{-9} \mathrm{M}$ rhodamine 6G (R6G) adsorbed on (a) graphene reduced by $\mathrm{NaBH}_{4}$, and (b) charcoal (activated) (lower wave number range). The spectra were acquired for $300 \mathrm{~s}$ for charcoal and $500 \mathrm{~s}$ for graphene with 1 accumulation.

However, for the white, non- fluorescent analyte, paracetamol solution, adsorbed on different carbon substrates, signals could be obtained up to $10^{-5} \mathrm{M}$ concentration (Figure 4). Raman signals were not observable beyond this concentration on any carbon substrates including graphene for this compound.

\section{Discussion}

From the results obtained it is apparent that chemically reduced graphene along with other carbon based materials used as substrates resulted in Raman signals for all the fluorescent analytes even at $10^{-7} \mathrm{M}$. The results obtained are akin to that obtained by Vosgröne et $a l^{36}$, where the authors have quantified the resonance effects of xanthenes dyes such as R6G using surface enhanced resonance Raman spectroscopy (SERRS). Furthermore, in the case where the excitation wavelength approached the electronic transition $\left(S_{0}-S_{1}\right)$ of the molecule, as was observed for R6G, Raman spectra at a concentration of $10^{-9} \mathrm{M}$ could be recorded on graphene as well as on charcoal (activated). On the other hand, with the non-fluorescent molecule, paracetamol, the Raman spectrum could only be obtained up to $10^{-5} \mathrm{M}$ concentration. Considering the underlying reasons for the above observation, firstly, it is clear that adsorption on carbon substrates reduced the fluorescence for R6G and RB and the spectra observed are more due to resonance effect of the excitation. In case of $\mathrm{CV}$ and NBA, the resonant effect did not play significant role as the excitation wavelength used for the study was 514.5, which was away from the absorbance maxima of these molecules. We believe that by using a wavelength in resonance with the electronic transition of these molecules would increase the sensitivity of detection. Secondly, carbonaceous materials are widely known as industrial adsorbents ${ }^{37}$. The adsorption processes are classified into two types, chemical (chemisorption) and physical (physisorption), depending on the nature of the interactive forces involved between the adsorbate and the adsorbent. In chemisorption, an atom or molecule is bound to the surface by the formation of some type of localized chemical bond. However, physisorption occurs as a result of relatively nonspecific intermolecular forces classified as van der Waals, London, and dispersion forces ${ }^{38}$. These nonspecific inter-molecular forces arise from the motion of electrons in molecules. The Raman signals observed for these molecules suggest that if chemical enhancement mechanism was attributing to the signal enhancement, one should, 


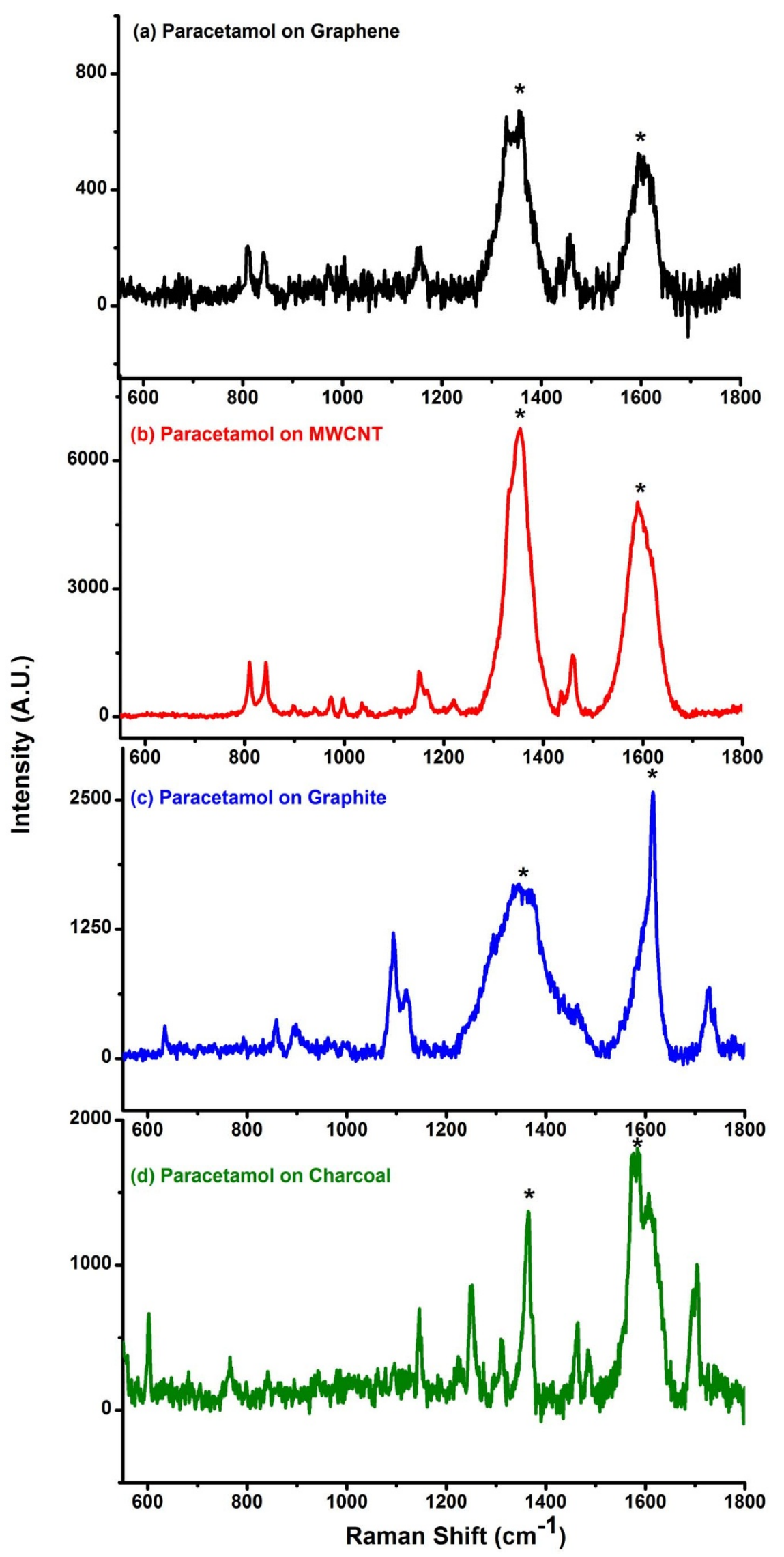

Figure $4 \mid$ Raman spectra of $10^{-5} \mathrm{M}$ Paracetamol adsorbed on (a) graphene reduced by NaBH4, (b) multi-walled carbon nanotube (MWCNT), (c) graphite and (d) charcoal (activated). (* represent carbon peaks). 
in principle, obtain enhanced signal for both the fluorescent and non-fluorescent compounds. It should be noted that in the case of paracetamol, a non-fluorescent molecule, Raman signals beyond $10^{-5} \mathrm{M}$ concentration could not be obtained for any carbon substrate including chemically reduced graphene.

In Summary, we have experimentally demonstrated the observation of enhanced Raman signals of various analytes adsorbed on various carbon substrates including chemically synthesized graphene. We have shown that the Raman signals were due to resonant effect and fluorescence suppression (in the case of fluorescent samples) owing to efficient adsorption on various carbon substrates. The enhanced signals observed at $10^{-9} \mathrm{M}$ concentrations for R6G is more due to the resonance effect rather than surface enhancement due to carbon substrates. Thus, we have demonstrated that the chemically synthesized graphene is "really" not a unique substrate for observing surface enhanced Raman signals. We hasten to add that these results and conclusions do not preclude the possibility of Raman enhancement or fluorescence quenching from single layer graphene. However, the results described here including the data from multiwalled carbon nanotubes indicate that the possibility of Graphene induced surface enhanced Raman effect and fluorescence quenching effect is an open question.

\section{Methods}

In our experiment we have used some common fluorescent molecules such as R6G, $\mathrm{RhB}, \mathrm{CV}, \mathrm{NBA}$ and non fluorescence molecule paracetamol for Raman probe.

Preparation of GO. The graphite oxide was synthesized from graphite powder following the Hummers method. Typically, concentrated $\mathrm{H}_{2} \mathrm{SO}_{4}(69 \mathrm{~mL})$ was added to a mixture of graphite powder $(3.0 \mathrm{~g})$ and $\mathrm{NaNO}_{3}(1.5 \mathrm{~g})$ and the mixture was cooled to $0^{\circ} \mathrm{C}$. $\mathrm{KMnO}_{4}(9.0 \mathrm{~g})$ was added slowly in portions to keep the reaction temperature below $20^{\circ} \mathrm{C}$. The reaction was warmed to $35^{\circ} \mathrm{C}$ and stirred for $30 \mathrm{~min}$, at which time water $(138 \mathrm{~mL})$ was added slowly, resulting in an exothermic reaction and the temperature rose to $98^{\circ} \mathrm{C}$. External heating was introduced to maintain the reaction temperature at $98^{\circ} \mathrm{C}$ for $15 \mathrm{~min}$, then the heating was removed and the reaction was cooled using water bath for $10 \mathrm{~min}$. Additional water $(420 \mathrm{~mL})$ and $30 \%$ $\mathrm{H}_{2} \mathrm{O}_{2}(3 \mathrm{~mL})$ were added, producing another exotherm and to produce bright yellow precipitate. This mixture was cooled to room temperature and centrifuged at $4000 \mathrm{rpm}$. This yielded a brownish-yellow precipitate of GO which was washed several times with $5 \% \mathrm{HCl}$ and then with water/ethanol and finally dried in air. $\mathrm{GO}$ obtained was subjected to ultrasonication for $40 \mathrm{~min}$ ( $20 \%$ amplitude) in order to exfoliate into graphene oxide in ultrapure water. Homogeneous dispersion of GO $\left(1 \mathrm{mgmL}^{-1}\right)$ obtained was directly used for reduction to get reduced GO nanosheets.

i) Synthesis of Graphene by reduction of GO by Sodium Borohydride. GO suspension $(100 \mathrm{~mL})$ was taken in a conical flask. The $\mathrm{pH}$ of this solution was adjusted to $9-10$ by liquid ammonia solution. Sodium borohydride (reagent grade, 98.5\%, Sigma-Aldrich) was directly added under magnetic stirring, and the mixture was kept at $80^{\circ} \mathrm{C}$ for $1 \mathrm{~h}$ with constant stirring. The brown dispersion of GO changed to black in colour upon reduction with $\mathrm{NaBH}_{4}$ The reduction product was separated by filtration and washed with large amounts of water several times to remove most residual ions. The reduced GO was kept in vacuum desiccators.

ii) Synthesis of Graphene by reduction of GO by using clove extract. Clove Extract was prepared by soaking $25 \mathrm{~g}$ of clove in $500 \mathrm{~mL}$ of water for $24 \mathrm{hr}$. Then it was filtered and used directly as reducing agent. GO $(100 \mathrm{~mL})$ suspension was taken in a conical flask. The $\mathrm{pH}$ of this solution was adjusted to $9-10$ by liquid ammonia solution. Clove extract $(200 \mathrm{~mL})$ was added under magnetic stirring and mixture was kept at $80-90^{\circ} \mathrm{C}$ for $6 \mathrm{hrs}$. Reaction was monitored by UV-Vis spectroscopy. The reduction product was separated by filtration and washed with large amounts of water several times. The product, graphene was confirmed using IR and XRD studies [Details of the characterisation of graphene are provided in Supplementary Information].

Graphite, MWCNT and activated charcoal were purchased commercially and used for experiments without further treatment. Charcoal, MWCNT and Graphite solutions $\left(1 \mathrm{mgmL}^{-1}\right)$ were prepared by dispersing the carbon substrates in milliQ water followed by sonication for 2 hours. Rhodamine $6 \mathrm{G}$ (R6G), Rhodamine B $(\mathrm{RhB})$, Nile Blue A (NBA) and Acetaminophen (paracetamol) were procured from Sigma-Aldrich while Crystal Violet (CV) was purchased from Sinopharm Chemical Reagent Co., Ltd. and were used as received. The solutions of the analytes were also prepared in milliQ water and different concentrations were prepared by serial dilution method. Equal ratios of the analyte and the carbon substrates were taken and mixed physically by vortexing for the Raman measurements. Nitrogen adsorption-desorption isotherms were recorded at $-196^{\circ} \mathrm{C}$ by using Micromeritics surface area analyzer model ASAP 2020. The specific surface area was calculated using Brunauer-Emmett-Teller (BET) method in the relative pressure $\left(\mathrm{p} / \mathrm{p}_{0}\right)$ range $0.05-0.25$ from adsorption branch of the isotherm.
The pore size distribution was calculated by Barrett-Joyner-Halenda (BJH) method from the desorption branch.

Raman spectroscopic experiments. Raman experiments were conducted using Renishaw's InVia Raman Microscope with an Ar ion laser with excitation wavelength of $514.5 \mathrm{~nm}$ with a $50 \times \mathrm{L}$ objective. The laser power on the sample was kept at $0.3 \mathrm{~mW}$ to $3 \mathrm{~mW}$ and the spectra were acquired for $10 \mathrm{~s}$ with 1 accumulation unless mentioned otherwise.

1. Neto, A. H. C., Guinea, F., Peres, N. M. R., Novoselov, K. S. \& Geim, A. K. The electronic properties. Rev. Mod. Phys. 81, 109-162 (2009).

2. Ferrari, A. C. et al. Raman spectrum of graphene and graphene layers. Phy. Rev. Lett. 97, 187401-187404 (2006).

3. Avouris, P. Graphene: Electronic and Photonic Properties and Devices. Nano Lett. 10, 4285-4294 (2010)

4. Cui, X., Zhang, C., Hao, R. \& Hou, Y. Liquid-phase exfoliation, functionalization and applications of graphene. Nanoscale 3, 2118-2126 (2011).

5. Ling, X. et al. Can Graphene be used as a Substrate for Raman Enhancement? Nano Lett. 10, 553-561 (2010).

6. Yu, X. et al. Tuning Chemical Enhancement of SERS by Controlling the Chemical Reduction of Graphene Oxide Nanosheets. ACS Nano. 5, 952-958 (2011).

7. Sun, S. \& Wu, P. Competitive surface-enhanced Raman scattering effects in noble metal nanoparticle-decorated graphene sheets. Phys. Chem. Chem. Phys. 13, 21116-21120 (2011).

8. Yaghobian, F., Korn, T. \& Schuller, C. Frequency Shift in Graphene-Enhanced Raman Signal of Molecules. Chem. Phys. Chem. 13, 4271-4276 (2012).

9. Qiu, C. et al. Investigation of n-Layer Graphenes as Substrates for Raman Enhancement of Crystal Violet. J. Phys. Chem. C 115, 10019-10025 (2011)

10. Bruna, M. \& Borini, S. Optical constants of graphene layers in the visible range. Appl. Phys. Lett. 94, 031901(1-3) (2009).

11. Rana, F. Graphene terahertz plasmon oscillators. IEEE Trans. Nanotechnol. 7, 91-99 (2008).

12. Grigorenko, A. N., Polini, M. \& Novoselov, K. S. Grapheneplasmonics. Nature photonics 6, 749-758 (2012).

13. Xie, L., Ling, X., Fang, Y., Zhang, J. \& Liu, Z. Graphene as a Substrate to Suppress Fluorescence in Resonance Raman Spectroscopy. J. Am. Chem. Soc. 131, 9890-9891 (2009).

14. Xu, W. et al. Surface enhanced Raman spectroscopy on a flat graphene surface. PNAS 109, 9281-9286 (2012)

15. Ritchie, G. \& Burstein, E. Luminescence of dye molecules adsorbed at an $\mathrm{Ag}$ surface. Phys. Rev. B 24, 4843-4846 (1981).

16. Tanimura, K., Kawal, T. \& Sakata, T. Electronic Structure of a Porphyrin Solid Film and Energy Transfer at the Interface with a Metal Substrate. J. Phys. Chem. 84, 751-756 (1980).

17. Ling, X., Wu, J., Xie, L. \& Zhang, J. Graphene-Thickness-Dependent GrapheneEnhanced Raman Scattering. J. Phys. Chem. C 117, 2369-2376 (2013).

18. Thrall, E. S., Crowther, A. C., Yu, Z. \& Brus, L. E. R6G on Graphene: High Raman detection Sensitivity, Yet Decreased Raman Cross-section. Nano Lett. 12, 1571-1577 (2012).

19. Liu, Z. B. et al. Porphyrin and fullerene covalently functionalized graphene hybrid materials with large nonlinear optical properties. J. Phys. Chem. B 113, 9681-9686 (2009).

20. Alvaro, M., Atienzar, P., Bourdelande, J. L. \& Garcia, H. An organically modified single wall carbon nanotube containing pyrene chromophore: fluorescence and diffuse reflectance laser flash photolysis study. Chem. Phys. Lett. 384, 119-123 (2004).

21. Liang, W., Chen, X., Sa, Y., Feng, Y., Wang, Y. \& Lin, W. Graphene oxide as a substrate for Raman enhancement. Appl Phys A 109, 81-85 (2012).

22. Konkena, B. \& Vasudevan, S. Resonance Raman Detection and Estimation in the Aqueous Phase Using Water Dispersible Cyclodextrin: Reduced-Graphene Oxide Sheets. Anal. Chem. 85, 5114-5119 (2013)

23. Ramakrishna Matte, H. S. S., Subrahmanyam, K. S., Venkata Rao, K., George, S. J. \& Rao, C. N. R. Quenching of fluorescence of aromatic molecules by graphene due to electron transfer. Chem. Phys. Lett. 506, 260-264 (2011).

24. Swathi, R. S. \& Sebastian, K. L. Resonance Energy Transfer from a Dye Molecule to Graphene. J. Chem. Phys. 129, 054703(1-9) (2009).

25. Swathi, R. S. \& Sebastian, K. L. Long range resonance energy transfer from a dye molecule to graphene has (distance)-4 dependence. J. Chem. Phys. 130, 086101 (1-3) (2009).

26. Biswas, N. \& Umapathy, S. Partitioning of the total reorganization energy into its vibrational and solvent (inertial and reorientational) contributions using resonance Raman intensities. Chem. Phys. Lett. 294, 181-190 (1998).

27. Umapathy, S., Mallick, B. \& Lakshmanna, A. Mode-dependent dispersion in Raman line shapes: Observation and implications from ultrafast Raman loss spectroscopy. J. Chem. Phys. 133, 024505-(1-6) (2010).

28. Kagan, M. R. \& McReery, R. L. Reduction of Fluorescence Interference in Raman Spectroscopy via Analyte Adsorption on Graphitic Carbon. Anal. Chem. 66, 4159-4165 (1994).

29. Hummers, W. \& Offeman, R. Preparation of Graphitic Oxide. J. Am. Chem. Soc. 80, 1339-1339 (1958).

30. Vernekar, A. A. \& Mugesh, G. Hemin-Functionalized Reduced Graphene Oxide Nanosheets Reveal Peroxynitrite Reduction and Isomerization Activity. Chem. Eur. J. 18, 15122-15132 (2012). 
31. Si, Y. \& Samulski, E. T. Synthesis of Water Soluble Graphene. Nano Lett. 8, 1679-1682 (2008).

32. Ferrari, A. C. Raman spectroscopy of graphene and graphite: Disorder, electronphonon coupling, doping and non-adiabatic effects. Solid State Comm. 143, 47-57 (2007).

33. Malard, L. M., Pimenta, P. A., Dresselhaus, G. \& Dresselhaus, M. S. Raman spectroscopy in graphene. Phys. Rep. 473, 51-87 (2009).

34. Hildebrandt, P. \& Stockburger, M. Surface-Enhanced Resonance Raman Spectroscopy of Rhodamine 6G Adsorbed on Colloidal Silver. J. Phys. Chem. 88, 5935-5944 (1984).

35. Watanabe, H., Hayazawa, N., Inouye, Y. \& Kawata, S. DFT Vibrational Calculations of Rhodamine 6G Adsorbed on Silver: Analysis of Tip-Enhanced Raman Spectroscopy. J. Phys. Chem. B, 109, 5012-5020 (2005).

36. Vosgröne, T. \& Meixner, A. J. Surface- and Resonance-Enhanced Micro-Raman Spectroscopy of Xanthene Dyes: From the Ensemble to Single Molecules. ChemPhysChem 6, 154-163 (2005).

37. Rouquerol, F., Rouquerol, J. \& Sing, K. Adsorption by powders and porous solids: principles, methodology and applications, Academic Press, ISBN 0-12-598920-2 (1999).

38. Arnett, E. M., Hutchinson, B. J. \& Healy, M. H. Carbonaceous Solids as a Model for Adsorption by Dispersion Forces. J. Am. Chem. Soc. 110, 5255-5260 (1988).

\section{Acknowledgments}

The authors gratefully acknowledge Department of Science \& Technology, Council of Scientific \& Industrial Research, Indian Institute of Science, Bangalore and DRDO for their financial support. Authors gratefully acknowledge Prof Munichandraiah, IPC Department for allowing us to perform the BET surface area measurements of carbon materials. S.U. acknowledges the J.C. Bose fellowship from DST.

\section{Author contributions}

S.S. designed the experiments, analyzed the data and wrote the manuscript; N.K. carried out the synthesis of graphene and characterization; S.A. carried out the synthesis and Raman experiments; S.U. conceptualised the research programme and coordinated the work and contributed to the text. All authors read and approved the manuscript.

\section{Additional information}

Supplementary information accompanies this paper at http://www.nature.com/ scientificreports

Competing financial interests: The authors declare no competing financial interests.

How to cite this article: Sil, S., Kuhar, N., Acharya, S. \& Umapathy, S. Is Chemically Synthesized Graphene 'Really' a Unique Substrate for SERS and Fluorescence Quenching? Sci. Rep. 3, 3336; DOI:10.1038/srep03336 (2013).

(c) $($ ) $\Theta$ This work is licensed under a Creative Commons AttributionBY NC ND NonCommercial-NoDerivs 3.0 Unported license. To view a copy of this license, visit http://creativecommons.org/licenses/by-nc-nd/3.0 